\title{
Community-acquired methicillin-resistant Staphylococcus aureus carrying SCCmec type IV in southern Brazil
}

\author{
Luciane Cristina Gelatti ${ }^{[1],[2], ~ R e n a n ~ R a n g e l ~ B o n a m i g o ~}{ }^{[1]}$, Fernanda Matsiko Inoue ${ }^{[3]}$, \\ Mirian Silva do Carmo ${ }^{[3]}$, Ana Paula Becker ${ }^{[1]}$, Fernanda Marques da Silva Castrucci ${ }^{[3]}$, \\ Antônio Carlos Campos Pignatari ${ }^{[3]}$ and Pedro Alves d' Azevedo ${ }^{[1]}$
}

[1]. Laboratório de Cocos Gram-positivos, Universidade Federal de Ciências da Saúde de Porto Alegre, Porto Alegre, RS. [2]. Faculdade Serra da Mesa, Uruaçu, GO. [3]. Laboratório Especial de Microbiologia Clínica, Universidade Federal de São Paulo, São Paulo, SP.

\begin{abstract}
Introduction: Methicillin-resistant Staphylococcus aureus (MRSA) is an important pathogen commonly associated with nosocomial infections. However, it has also been associated with community-acquired skin and soft tissue infections (CA-MRSA). There are few data on the identification and prevalence of CA-MRSA infections in Brazil. Methods: This is a cross-sectional study of 104 patients with community-acquired skin infections attending two health care centers in Porto Alegre, southern Brazil. MRSA isolates were characterized by molecular methods, including detection of the mecA gene by PCR, gene SCCmec typing, PantonValentine leukocidin (PVL) detection, pulsed-field gel electrophoresis (PFGE), and multilocus sequence typing (MLST). Results: From the 104 samples, 58 Staphylococcus aureus isolates were obtained, of which five (8.6\%) had a CA-MRSA-resistant profile. All five isolates had the mecA gene and amplified to SCCmec type IV. Analysis of chromosomal DNA by PFGE revealed the presence of two clusters related to international clones (OSPC and USA 300), with a Dice similarity coefficient $>80 \%$. The study was complemented by MLST, which detected three different strains: ST30, ST8, and ST45, the latter not presenting any relation with the clones compared in PFGE. Conclusions: The presence of CA-MRSA reveals an important change in the epidemiology of this pathogen and adds new elements to the knowledge of the molecular biology of infections by MRSA with SCCmec type IV in southern Brazil.
\end{abstract}

Keywords: Staphylococcus aureus. Methicillin-resistant. Skin infections. Soft tissue infections.

\section{INTRODUCTION}

Staphylococcus aureus is a microorganism responsible for a wide variety of infectious diseases. The major concern refers mainly to methicillin-resistant Staphylococcus aureus (MRSA) isolates, which traditionally were limited to the hospital (HA) ${ }^{1}$. However, in recent years, community-acquired methicillinresistant Staphylococcus aureus (CA-MRSA) infections have been reported worldwide ${ }^{2-5}$. CA-MRSA isolates frequently cause infections involving the skin and soft tissues, such as cellulites and abscesses, but they might be responsible for severe infections, such as necrotizing pneumonia and fasciitis, meningitis, bacteremia, and septic shock ${ }^{6-9}$.

Infections by CA-MRSA isolates are usually associated with children, young adults, and healthy people, particularly those living close together or having close physical contact with each other. Most reports in the literature refer to athletes of team sports, individuals doing military service, prison inmates, intravenous drug users, homeless people, and children in day

Address to: Dra. Luciane Cristina Gelatti. Laboratório de Cocos Gram-positivos/ UFCSPA. Rua Sarmento Leite 245/Sala 203, 90050-170 Porto Alegre, RS, Brasil. Phone: 5551 3303-9000

e-mail: lucianegelatti@hotmail.com

Received in 09/08/2011

Accepted in 30/09/2011 care centers as having a higher risk of developing CA-MRSA infections. The typical presentation would be a young athlete with abscess and cellulites, with contributing factors such as physical contact, skin lesions, and sharing of contaminated equipment ${ }^{10-13}$.

Epidemiologically, clinical MRSA isolates are defined as CA-MRSA if collected from outpatients or collected up to 48 hours after hospital admission. However, the time criterion should not be considered in patients with risk factors for HA-MRSA. Recent hospitalization, use of antimicrobial drugs, surgical procedures, use of long-term indwelling catheter or other intravascular and cutaneous devices, and admission to nursing homes should be excluded ${ }^{14,15}$. A phenotypic profile of increased susceptibility to antimicrobial agents, except to beta-lactams, is a commonly observed characteristic. This manifests itself as a lack of other resistance genes, except for mecA, in the staphylococcal cassette chromosome mec (SCCmec) genomic complex of types IV and V representatives, usually associated with communityacquired infections. SCCmec types IV and V present a smaller resistance island when compared to the islands carried by isolated clones in nosocomial infections (SCCmec I, II, and III) ${ }^{16-18}$, which usually impart multiresistance to these isolates.

Panton-Valentine leukocidin (PVL) expression is another important genetic characteristic associated with CA-MRSA strains. PVL is encoded by $l u k-P V$ and $l u k S-P V$ genes, and its presence in Staphylococcus aureus isolates is associated with 
tissue necrosis and leukocyte destruction by forming pores in the cell membrane ${ }^{19}$. This exotoxin may be detected by searching for specific genes by through a polymerase chain reaction. CA-MRSA infections are the subject of much concern because the microorganism does not respond to the beta-lactam antimicrobial agents commonly used to empirically treat various community infections ${ }^{20}$.

The purpose of our study was to characterize Staphylococcus aureus isolates from community-acquired skin and soft tissue infections in the City of Porto Alegre while searching for CA-MRSA. There are few data on the identification and prevalence of CA-MRSA infections in Brazil.

\section{METHODS}

\section{Bacterial isolates}

A cross-sectional study was performed. Staphylococcus aureus was originated from skin infections of patients attending two reference health care centers in southern Brazil (Sanitary Dermatology Outpatient Clinic of Rio Grande do Sul and the Emergency Unit of the Complexo Hospitalar Santa Casa de Porto Alegre) between September 2007 and March 2008. In addition to the clinical diagnosis and the phenotypic and genotypic characteristics of the Staphylococcus aureus, the age and gender of patients were also evaluated. The inclusion criteria were: outpatients or patients admitted to the hospital for up to $48 \mathrm{~h}$ and presence of superficial cutaneous and soft tissue infections. The exclusion criteria were: presence of any underlying illness possibly predisposing to frequent hospitalizations or frequent visits to medical facilities, surgical interventions, presence of intravenous catheter or long-term indwelling intravascular or cutaneous devices, admission to nursing homes, and isolation from other organisms except for Staphylococcus aureus. And positive samples in duplicate.

\section{Sample collection}

After decontaminating the margins and surface of the lesion with physiological saline and $70 \%$ alcohol, biological material was collected with a sterile swab. The material was sent to the Microbiology Laboratory of the Federal University of Health Sciences of Porto Alegre, where the standard procedures were performed for bacterial isolation and identification of organisms present in the lesions.

\section{Phenotypic characterization}

The tests used for phenotypic characterization of the Staphylococcus aureus were Gram staining, growth and fermentation on mannitol agar, coagulase test, and DNase test agar. Staphylococcus aureus ATCC 25923 and Staphylococcus epidermidis ATCC 12228 were used as positive and negative control, respectively.

\section{Susceptibility test}

The antibiotic susceptibility of the strains against the antibiotics mentioned below was tested by using the KirbyBauer disk diffusion method. The results of susceptibility testing were interpreted according to accepted guidelines ${ }^{21}$. Disk susceptibility testing was carried out using cefoxitin $(30 \mu \mathrm{g})$, ciprofloxacin $(5 \mu \mathrm{g})$, clindamycin $(2 \mu \mathrm{g})$, chloramphenicol $(30 \mu \mathrm{g})$, erythromycin $(15 \mu \mathrm{g})$, gentamicin $(10 \mu \mathrm{g})$, linezolid $(30 \mu \mathrm{g})$, oxacillin $(1 \mu \mathrm{g})$, and trimethoprim-sulfamethoxazole $(25 \mu \mathrm{g})$. Resistance to macrolides and lincosamides was assessed by D-test, with a distance of $15-26 \mathrm{~mm}$ between the clindamycin $(2 \mu \mathrm{g})$ and erythromycin $(15 \mu \mathrm{g})$ disks, as previously described ${ }^{22}$. Staphylococcus aureus ATCC 25923 was used as quality control in the disk diffusion technique.

\section{Molecular characterization}

Complete molecular characterization (presence of the mecA gene, determination of SCCmec type, detection of the gene $l u k F$, PFGE, and MLST) was performed for the MRSA isolates. Gene $m e c$ A characterization and $\mathrm{SCC} m e c$ typing were performed by multiplex polymerase chain reaction (PCR) assay according to the protocol previously developed by Zhang et al. ${ }^{23}$. To detect the PVL-encoding gene, primers were synthesized from genomic DNA sequences of the GenBank database (GenBank access number AB006796) according to Figueiredo et al. ${ }^{24}$. Analysis of chromosomal deoxyribonucleic acid (DNA) of MRSA isolates was performed by pulsed-field gel electrophoresis (PFGE) according to the protocol established by the Centers for Disease Control and Prevention for Staphylococcus aureus molecular typing ${ }^{25}$. Multilocus sequence typing (MLST) was performed by following the procedures in previously published protocols ${ }^{26}$. The sequences of the seven housekeeping genes ( $\operatorname{arc}$, aroE, glpF, gmk, pta, tpi, and yqiL) were compared to existing sequences in the MLST database at http://www.mlst.net for the assignment of allelic numbers. Sequence types (ST) were assigned according to their allelic profiles.

\section{Ethical considerations}

The research was approved by the Ethics Committee in Research of the Complexo Hospitalar da Santa Casa de Porto Alegre and the Ethics Committee in Health Research of the Escola Pública de Saúde do Rio Grande do Sul. To take part in the study, each patient or his or her legal representative signed a free and informed consent form.

\section{RESULTS}

A total of 104 patients, mostly (70.1\%) males, were analyzed for a period of seven months. With regard to age, $10(9.6 \%)$ patients were children, $27(26 \%)$ were between 18 and 39 years old, and 67 (64.4\%) were aged 40 years or older.

Isolation of Staphylococcus aureus was obtained in 58 $(55.8 \%)$ patients with skin and soft tissue infections; five (8.6\%) S. aureus isolates were resistant to oxacillin and cefoxitin, having been characterized as MRSA. These isolates were associated with community-acquired infections based on the epidemiological characteristics and phenotypic profile of sensitivity. Other patients showed negative cultures or growth of different organisms. Table 1 shows the resistance profile to antimicrobials of the MRSA isolates. Most MRSA were shown to be multisusceptible, a phenotypic characteristic observed in isolates 
TABLE 1 - Phenotypic and genotypic properties of methicillin-resistant Staphylococcus aureus with SCCmec type IV.

\begin{tabular}{lccccc}
\hline Isolate & Isolation date & $\begin{array}{c}\text { Antibiogram } \\
\text { (resistance to) }\end{array}$ & $\begin{array}{c}\text { SCCmec } \\
\text { type }\end{array}$ & $\begin{array}{c}\text { PVL- } \\
\text { encoding gene }\end{array}$ & ST \\
\hline A31651 & 09 Oct 2007 & CFO, CLI, ERI, OXA & IV & + & 30 \\
A31652 & 08 Nov 2007 & CFO, CIP, ERI, OXA & IV & - & 8 \\
A31655 & 30 Oct 2007 & CFO, OXA & IV & + & 30 \\
A31656 & 22 Nov2007 & CFO, OXA & IV & + & 30 \\
AD554 & 12 Dec 2007 & CFO, CLO, OXA & IV & - & 45 \\
\hline
\end{tabular}

SCCmec: staphylococcal cassette chromosome mec; PVL: Panton-Valentine leukocidin; ST: sequence type; CFO: cefoxitin; CIP: ciprofloxacin; CLI: clindamycin; CLO: chloramphenicol; ERI: erythromycin; OXA: oxacillin.

characterized as community-acquired. MRSA isolates (A31651 and AD554) were obtained from two patients from the community (a 39-year-old male and a 54-year-old female, respectively) presenting with skin infections (furunculosis). Another isolate (A31652) was obtained from the skin infections of a 3 -year-old male who presented with chicken pox wounds.
In addition, MRSA isolates (A31655 and A31656) were obtained from two outpatients (a 1-month-old female and a 7-year-old male, respectively), the first presenting with ocular injury, and the second with injury caused by lower-limb trauma, which evolved to cellulitis, pneumonia, and sepsis. Following these strict criteria, previously suggested by Salgado et al. ${ }^{14}$ and the Centers for Disease Control and Prevention (CDC) ${ }^{15}$, these five isolate cases were defined as representing community-acquired infections (CA-MRSA).

Similarly to MRSA, all the characterized samples presented an amplification of the themecA gene in the PCR and amplified to SCCmec type IV by the Zhang et al. ${ }^{23}$ method. Among the type IV SCCmec samples, three presented an amplification of the PVL-encoding gene. The PFGE analysis of chromosomal DNA revealed two clusters related to international clones: OSPC (Oceania Southwest Pacific Clone) and USA300 (Figure 1), with a Dice similarity coefficient $>80 \%$. The study was supplemented with MLST, which detected three different strains: ST30, ST8, and ST45, the latter not presenting any relation with the clones compared in the PFGE.

Dice (opt:0.80\%) (Tol 2.0\%-2.0\%) (H>0.0\% S>0.0\%) [0.0\%-100.0\%]

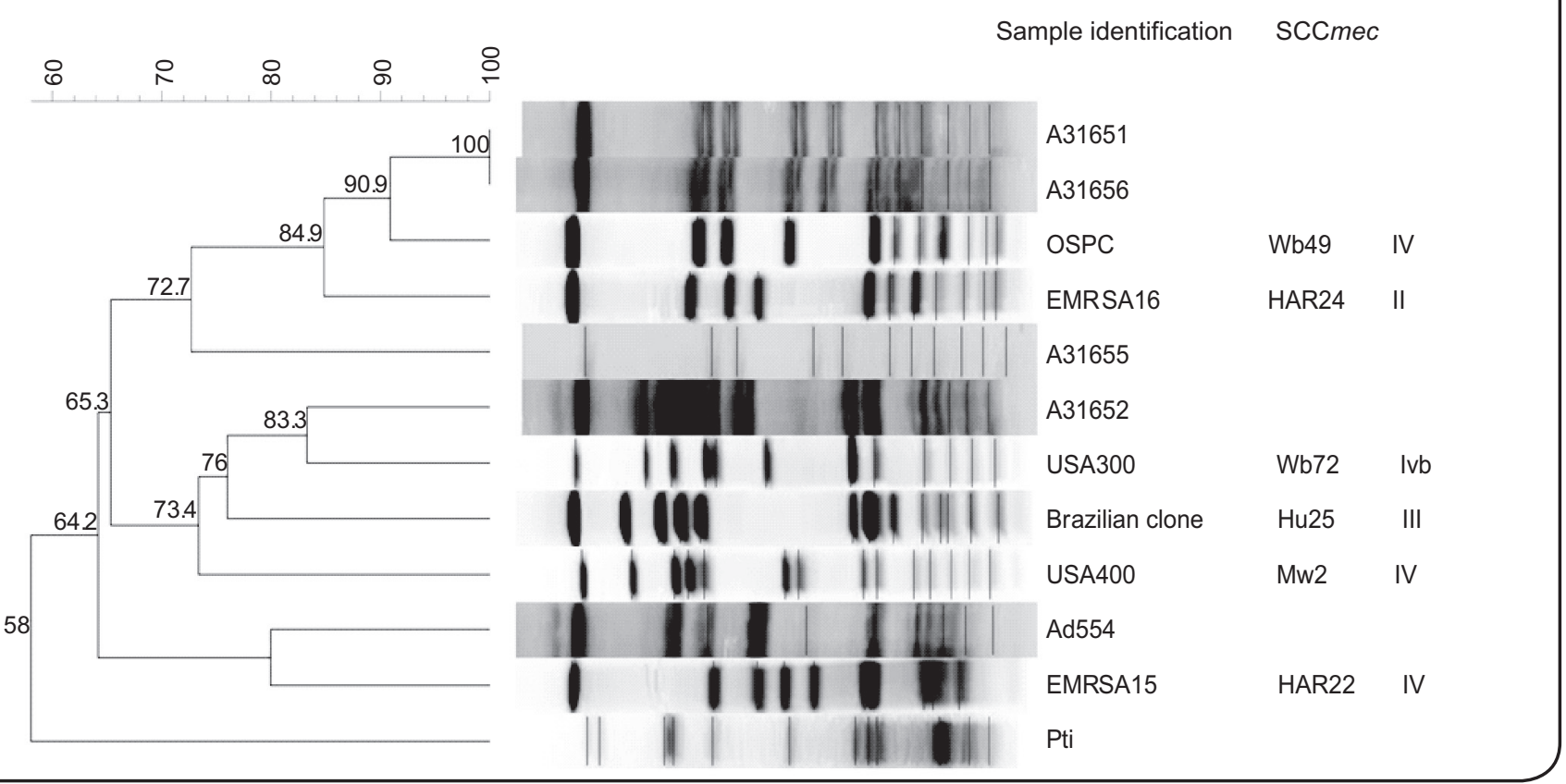

FIGURE 1 - Cluster analysis of CA-MRSA samples containing SCCmec type IV compared with international standard samples using Dice similarity coefficient.

SCCmec: staphylococcal cassette chromosome mec; CA-MRSA: community-acquired methicillin-resistant Staphylococcus aureus.

\section{DISCUSSION}

The present study allowed showing the presence of MRSA among Staphylococcus aureus isolates characterized as community-acquired (CA-MRSA in $8.6 \%$ of cases), indicating a change in the epidemiology of this organism, as resistant isolates were found outside a hospital setting. The findings confirm the potential emergence of MRSA in community settings in Porto
Alegre, Brazil, in individuals who do not have traditional risk factors for infection by this pathogen.

The first cases of CA-MRSA in Porto Alegre are from 2002 and $2003^{24}$, about ten years after the first reports of typical community-acquired MRSA in western Australia, which infected the local aboriginal population ${ }^{27}$. Later, another study showed the existence of this isolated MRSA in the southeast region (Rio de Janeiro) ${ }^{28}$. The fact that the two geographic areas 
(Porto Alegre and Rio de Janeiro) are separated by about 1,500 kilometers of distance should indicate that this isolated MRSA is found in different regions of the country.

Our study is pioneering in Brazil in identifying and characterizing isolated MRSA found only in skin and soft tissue infections. When compared with the results of studies from other countries, a great variation in the taxes of prevalence of CAMRSA is observed. However, an important observation in all the studies is that, since the organism was first found, the prevalence of infections caused by CA-MRSA has been increasing. Reports from other countries, such as the United States, show that there is a great variation in occurrences related to geography. In a study done on 11 emergency centers in different cities in the United States in August 2004, a global prevalence rate of 59\% was observed, and the variation found was between $15 \%$ and $74 \%{ }^{29}$. More recently, in a study done in 10 primary care clinics in Texas, United States, Forcade et al. ${ }^{30}$ detected a similar value: about $61 \%$ of skin and soft tissue infections caused by CA-MRSA.

A multicenter study conducted in Argentina showed a high prevalence of methicillin-resistant Staphylococus aureus isolated in infections of children from the community. Among the 447 samples analyzed in the years 2006 and 2007, 281 met the criteria set for CA-MRSA. This investigation showed that $62 \%$ of isolates came from skin and soft tissue infections ${ }^{31}$. In contrast, in the Nordic countries (Denmark, Finland, Iceland, Norway, and Sweden), the rates of infections by MRSA are less than $1 \%$; however, in all these countries a significant increase in the infection rate was observed in the last 3 to 5 years, possibly related to an alteration in the behavior of the isolated MRSA ${ }^{32}$.

The CA-MRSA isolates obtained in the present study showed an increased susceptibility profile to antimicrobial agents, although three isolates showed resistance to other antimicrobials, such as chloramphenicol (AD554), ciprofloxacin and erythromycin (A31652), and erythromycin and clindamycin (A31651). The results herein parallel those described by Ribeiro et al. ${ }^{28}$ and Chen et al..$^{33}$, who reported CA-MRSA strains resistant to other non-beta-lactam antimicrobials.

The molecular characterization showed the presence of clonal type IV in all MRSA isolates. The findings corroborate the increased susceptibility to antimicrobials, attributed to SCCmec type IV, which is smaller and usually does not aggregate other resistance determinants, unlike classical hospital strains. The analysis of chromosomal DNA revealed a similarity to the OSPC clone in two isolates. Of the two patients infected with CA-MRSA similar to the OSPC clone (SCCmec IV, ST30, PVL+), one developed severe necrotizing pneumonia (A31656), secondary to soft tissue lesions, and was hospitalized for 50 days. The second patient (A31651) infected with CA-MRSA of the same clone showed recurrent furunculosis and was treated with first-generation cephalosporin, which provided no cure for the infection and lesions. The isolates pertaining to this clone showed Panton-Valentine leukocidin-encoding genes, which contributed to aggravating the clinical status. The results confirm the findings of Ribeiro et al..$^{28}$ in isolates of skin infections in the same Brazilian city. The presence of circulating OSPC clones is alarming because an outbreak in the neighboring country, Uruguay, was attributed to this very clone, and several patients developed severe pneumonia ${ }^{34}$. Although presenting SCCmec IV, ST30, and PVL+, a third isolate (A31655) did not show any similarity with the OSPC clone.

Another international clone found in our study was USA300 (SCCmec IV, ST8, PVL-). Most isolates pertaining to the USA300 clone showed PVL-encoding genes ${ }^{18}$; however, recent studies have shown samples that do not produce the toxin ${ }^{28,29}$.

The USA300 clone has been reported in many regions of the United States, especially among football players and prison inmates ${ }^{12,31}$. A recent study in the United States has shown that $50 \%$ of MRSA isolates from skin and soft tissue infections belong to the USA300 clone $^{35}$.

We emphasize the importance of identification and therapeutic practice in infections associated with $S$. aureus, particularly those caused by methicillin-resistant isolates. In 1999, four children in the States of Minnesota and North Dakota in the United States died from infections caused by CA-MRSA $^{36}$. In these cases, cephalosporins were initially used in the treatment, and the delay in using an appropriate antimicrobial agent may have contributed to the outcome. Another study conducted in the United States showed that $80 \%$ of patients with skin and soft tissue infections received empirical treatment. Of these, $57 \%$ did not respond to treatment because the microorganism was resistant ${ }^{29}$. These findings suggest the need to reassess the choice of empirical treatments for skin and soft tissue infections in areas where MRSA is prevalent in the community. In a teaching hospital in California, United States, MRSA was detected in $87 \%$ of Staphylococcus aureus isolates from patients with skin infections treated at its emergency center. This study supports the drainage of skin abscesses as a preferred treatment as opposed to using antimicrobial agents ${ }^{37}$. Despite controversies concerning the choice of treatment and even of the antimicrobial agent for managing skin infections caused by $S$. aureus, cephalosporins are among those with the highest utilization rates.

The emergence of Staphylococcus aureus type IV as a pathogen associated with community-acquired skin and soft tissue infections is remarkable. Another important aspect that might prevent the dissemination of this microorganism is the certainty that these strains circulate outside the hospital setting. Thus, the importance of laboratory cultivation and antimicrobial profiling of clinical material from skin and soft tissue infections in primary care facilities, such as hospital emergency centers and dermatological care centers, is unequivocal. By identifying the microorganism and performing a susceptibility test, an appropriate treatment that focuses on control measures for this pathogen may be implemented.

We described the emergence of community-acquired methicillin-resistant Staphylococcus aureus carrying SCCmec type IV in two health care centers in Porto Alegre, southern Brazil. These observations provide some insight into the epidemiology of this pathogen, which is of clinical importance, and add new elements to our understanding of the molecular biology of infections by CA-MRSA in our country. 


\section{CONFLICT OF INTEREST}

The authors declare that there is no conflict of interest.

\section{REFERENCES}

1. Vandenesch F, Naimi T, Enright MC, Lina G, Nimmo GR, Heffernan H, et al. Community-acquired methicillin-resistant Staphylococcus aureus carrying Panton-Valentine leukocidin genes: worldwide emergence. Emerg Infect Dis 2003; 9:978-984.

2. Salmenlinna S, Lyytikainen O, Vuopio-Varkila J. Community-acquired methicillin-resistant Staphylococcus aureus, Finland. Emerg Infect Dis 2002; 8:602-607.

3. Miklasevics E, Haeggman S, Balode A, Sanchez B, Martinsons A, OlssonLiljequist B, et al. Report on the first PVL-positive community acquired MRSA strain in Latvia. Euro Surveill 2004; 9:29-30.

4. Chen CJ, Huang YC, Chiu CH, Su LH, Lin TY. Clinical features and genotyping analysis of community-acquired methicillin-resistant Staphylococcus aureus infections in Taiwanese children. Pediatr Infect Dis J 2005; 24:40-45.

5. Nevet A, Ashkenazi S, Samra Z, Livni G. Community-associated methicillinresistant Staphylococcus aureus infections in Israel. Isr Med Assoc J 2010; 12:428-430.

6. Diep BA, Gill SR, Chang RF, Phan TH, Chen JH, Davidson MG, et al. Complete genome sequence of USA300, an epidemic clone of community-acquired methicillin-resistant Staphylococcus aureus. Lancet 2006; 367:731-739.

7. John CC, Schreiber JR. Therapies and vaccines for emerging bacterial infections: learning from methicillin-resistant Staphylococcus aureus. Pediatr Clin North Am 2006; 53:699-713.

8. Dickson RP, Martinez SM, Ortiz JR. A case of rapidly progressive necrotizing pneumonia caused by community-acquired methicillin-resistant Staphylococcus aureus. Respir Care 2008; 53:1223-1226.

9. Naesens R, Ronsyn M, Druwé P, Denis O, Leven M, Jeurissen A. Central nervous system invasion by community-acquired meticillin-resistant Staphylococcus aureus. J Med Microbiol 2009; 58:1247-1251.

10. Dietrich DW, Auld DB, Mermel LA. Community-acquired methicillin-resistant Staphylococcus aureusin southern New England children. Pediatrics 2004; 113:347-352

11. Zinderman CE, Conner B, Malakooti MA, LaMar JE, Armstrong A, Bohnker BK. Community-acquired methicillin-resistant Staphylococcus aureus among military recruits. Emerg Infect Dis 2004; 10:941-944.

12. Nguyen DM, Mascola L, Bancroft E. Recurring methicillin-resistant Staphylococcus aureus infections in a football team. Emerg Infect Dis 2005; 11:526-532.

13. Kluytmans-Vandenbergh MF, Kluytmans JA. Community-acquired methicillinresistant Staphylococcus aureus: current perspectives. Clin Microbiol Infect 2006; 12 (supl I): 9-15.

14. Salgado CD, Farr BM, Calfee DP. Community acquired methicillin-resistant Staphylococcus aureus: a meta-analysis of prevalence and risk factors. Clin Infect Dis 2003; 36:131-139.

15. Centers for Disease Control and Prevention. Community-associated MRSA information for clinicians. [Cited 2007 January 8]. Available from: http://www. cdc.gov/ncidod/dhqp/ar_mrsa_ca_clinicians.html\#4/.

16. Amorim ML, Sousa MA, SanchesI S, Sá-Leão R, Cabeda JM, Amorim JM, et al. Clonal and antibiotic resistance profiles of methicillin-resistant Staphylococcus aureus (MRSA) from a Portuguese hospital over time. Microb Drug Resist 2002; 8:301-309.

17. Pérez-Roth E, Lorenzo-Díaz F, Batista N, Moreno A, Méndez-Álvarez S. Tracking methicillin-resistant Staphylococcus aureus clones during a 5-years period (1998 to 2002) in a Spanish hospital. J Clin Microbiol 2004; 42:4649-4656.

18. Tenover FC, McDougal LK, Goering RV, Killgore G, Projan SJ, Patel JB, et al. Characterization of a strain of community-associated methicillin-resistant Staphylococcus aureus widely disseminated in the United States. J Clin Microbiol 2006; 44:108-118.

19. DufourP, GilletY,Bes M, Lina G, VandeneschF, FloretD, etal. Community-acquired methicillin-resistant Staphylococcus aureus infection in France: emergence of a single clone that produces Panton-Valentine leukocidin. Clin Infect Dis 2002; 35:819-824.

20. Diep BA, Sensabaugh GF, Somboona NS, Carleton HA, Perdreau-Remington F. Widespread skin and soft-tissue infections due to two methicillin-resistant Staphylococcus aureus strains harboring the genes for Panton-Valentine leucocidin. J Clin Microbiol 2004; 42:2080-2084.

21. Clinical and Laboratory Standards Institute. Performance Standards for Antimicrobial Disk Susceptibility Tests. Approved Standard. $9^{\text {th }}$ ed. M2-A8. Pennsylvania: Clinical and Laboratory Standards Institute; 2007.

22. Fiebelkorn KR, Crawford SA, McElmeel ML, Jorgensen JH. Practical disk diffusion method for detection of inducible clindamycin resistance in Staphylococcus aureusand coagulase negative staphylococci. J Clin Microbiol 2003; 41:4740-4744

23. Zhang K, McClure JA, Elsayed S, Louie T, Conly JM. Novel multiplex PCR assay for characterization and concomitant subtyping of staphylo-coccal cassette chromosome mec types I to V in methicillin-resistant Staphylococcus aureus. J Clin Microbiol 2005; 43:5026-5033.

24. Ribeiro A, Dias C, Silva-Carvalho MC, Berquo L, Ferreira FA, Santos RN, et al. First report of infection with community-acquired methicillin-resistant Staphylococcus aureus in South America. J Clin Microbiol 2005; 43: 1985-1988.

25. McDougal LK, Steward CD, Killgore GE, Chaitram JM, McAllister SK, Tenover FC. Pulsed-field gel electrophoresis typing of oxacillin-resistant Staphylococcus aureus isolates from the United States: establishing a national database. J Clin Microbiol 2003; 41:5113-5120.

26. Enright MC, Day NPJ, Davies CE, Peacock SJ, Spratt BG. Multilocus sequence typing for characterization of methicillin-resistant and methicillin-susceptible clones of Staphylococcus aureus. J Clin Microbiol 2000; 38:1008-1015.

27. Udo EE, Pearman JW, Grubb WB. Genetic analysis of community isolates of methicillin-resistant Staphylococcus aureus in Western Australia. J Hosp Infect 1993; 25:97-108.

28. Ribeiro A, Coronado AZ, Silva-Carvalho MC, Ferreira-Carvalho BT, Dias C, Rozenbaum R, et al. Detection and characterization of international communityacquired infections by methicillin-resistant Staphylococcus aureus clones in Rio de Janeiro and Porto Alegre cities causing both community-and hospitalassociated diseases. DiagnMicrobiol Infect Dis 2007; 59:339-345.

29. Moran GJ, Krishnadasan A, Gorwitz RJ, Fosheim GE, McDougal LK, Carey RB, et al. Methicillin-resistant $S$. aureus infections among patients in the emergency department. N Engl J Med 2006; 355:666-674.

30. Forcade NA, Parchman ML, Jorgensen JH, Liem CD, Nyren NR, Treviño LB, et al. Prevalence, Severity, and Treatment of Community-Acquired MethicillinResistantStaphylococcus Aureus(CA-MRSA) Skin and Soft Tissue Infections in 10 Medical Clinics in Texas: A South Texas Ambulatory Research Network (STARNet) Study. J Am Board Fam Med 2011; 24:543-550.

31. Paganini H, DellaLatta MP, Opet BM, Ezcurra G,Uranga M, Aguirre C, et al. Estudio multicéntrico sobre las infecciones pediátricas por Staphylococcus aureus meticilino-resistente provenientes de la comunidad en la Argentina.Arc Argen Pediatr 2008; 106:397-403.

32. Skov R, SSAC MRSA Working Party. MRSA infections increasing in the Nordic countries. Euro Surveill 2005; 10(8):E050804.2.

33. Chen CJ, Huang YC, Chiu CH, Su LH, Lin TY. Clinical features and genotyping analysis of community-acquired methicillin-resistant Staphylococcus aureus infections in Taiwanese children. Pediatr Infect Dis J 2005; 24:40-45.

34. Ma XX, Galiana A, Pedreira W, Mowszowicz M, Chrisphersen I, Machiavello S, et al. Community-acquired methicillin-resistant Staphylococcus aureus, Uruguay. EmergInfec Dis 2005; 11:973-976.

35. Kazakova SV, Hageman JC, Matava M, Srinivasan A, Phelan L, Garfinkel B, et al. A clone of methicillin-resistant Staphylococcus aureus among professional football players. N Engl J Med 2005; 352:468-475.

36. Centers for Disease Control and Prevention. Four pediatric deaths from community-acquired methicillin-resistant Staphylococcus aureus-Minnesota and North Dakota 1997-1999. MMWR 1999; 48:707-710.

37. Rajendran PM, Young D, Maurer T, Chambers H, Perdreau-Remington F, Ro P, et al. Randomized, double-blind, placebo-controlled trial of cephalexin for treatment of uncomplicated skin abscesses in a population at risk for communityacquired methicillin-resistant Staphylococcus aureus infection. Antimicrob Agents Chemother 2007; 51:4044-4048. 